\title{
Using Quartz Crystal Microbalance for Field Measurement of Liquid Viscosities
}

\author{
Qingsong Bai and Xianhe Huang \\ School of Automation Engineering, University of Electronic Science and Technology of China, Chengdu 611731, China \\ Correspondence should be addressed to Xianhe Huang; xianhehuang@uestc.edu.cn
}

Received 25 November 2015; Revised 20 January 2016; Accepted 20 January 2016

Academic Editor: Vincenzo Spagnolo

Copyright (C) 2016 Q. Bai and X. Huang. This is an open access article distributed under the Creative Commons Attribution License, which permits unrestricted use, distribution, and reproduction in any medium, provided the original work is properly cited.

\begin{abstract}
The field measurement of liquid viscosities, especially the high viscous liquids, is challenging and often requires expensive equipment, long processing time, and lots of reagent. We use quartz crystal microbalances (QCMs) operating in solution which are also sensitive to the viscosity and density of the contacting solution. QCMs are typically investigated for sensor applications in which one surface of QCM completely immersed in Newtonian liquid, but the viscous damping in liquids would cause not only large frequency shifts but also large losses in the quality factor $Q$ leading to instability and even cessation of oscillation. A novel mass-sensitivity-based method for field measurement of liquid viscosities using a QCM is demonstrated in this paper and a model describing the influence of the liquid properties on the oscillation frequency is established as well. Two groups of verified experiments were performed and the experimental results show that the presented method is effective and possesses potential applications.
\end{abstract}

\section{Introduction}

The measurement of liquid viscosities is one of the most important steps in a variety of research and industrial fields. There are many techniques including capillary [1], falling body [2], oscillating [3], and ultrasonic [4] methods that can measure the viscosity of liquids. However, these commercial devices are usually costly, time consuming, and limited in the measurement range.

During the last few decades, quartz crystal microbalances (QCMs) are increasingly being studied as a sensitive, rapid, high-performance, and inexpensive microsensors capable of performing in liquid environments [5-12]. A QCM is a shear mode device consisting of a thin quartz disk sandwiched between two circular, metallic electrodes of the same diameters. The electrodes are used to excite the resonator whose frequency is inversely proportional to the thickness of the quartz plate. As a highly sensitive mass sensor, QCMs have been used in various areas of science and technology, such as mass detectors in vapors or solutions [13-16], biosensors $[17,18]$, and electrochemical microbalances or nanobalances [19-21].
The linear relationship between the mass change and the resonance frequency shift of the QCM is described by the Sauerbrey equation [22]:

$$
\Delta f=-\frac{2 f_{0}^{2}}{A \sqrt{\rho_{q} u_{q}}} \Delta m
$$

where $\Delta m$ and $\Delta f$ are mass change and frequency shift, respectively; $f_{0}$ is the operating frequency of the QCM; $A$ is the active area of the QCM electrodes; and $\rho_{q}$ and $u_{q}$ are the density and shear modulus of the piezoelectric quartz crystal, respectively.

It should be noted here that the Sauerbrey equation is established on the assumption that the mass change attached on the QCM surface is a rigid and even thin film. So the linear relation between the adsorbed mass and the change in frequency is not necessarily valid for viscoelastic films. Therefore, the Sauerbrey equation is invalid for the measurement of liquid parameters.

Kanazawa and Gordon II offered an additional method to measure Newtonian liquid using a QCM, namely, the total immersion of one surface of the QCM in sample liquid, and determined the behavior of the crystal/fluid system by 
examining the coupling of the elastic shear waves in the crystal to the viscous shear weaves in the sample liquid. In this manner, the resonance condition derives directly from the matching of appropriate boundary conditions on the shear waves. Thus, the Kanazawa model that reveals the relative relationship between the frequency shift of the QCM and the density and viscosity of the sample liquid has been developed [23]:

$$
\Delta f=f_{0}^{3 / 2}\left(\frac{\eta \rho}{\pi \rho_{q} u_{q}}\right)^{1 / 2}
$$

where $f_{0}$ is the operating frequency of the QCM; $\rho$ and $\eta$ are the density and viscosity of the sample liquid, respectively; $\rho_{q}$ and $u_{q}$ are the density and shear modulus of the quartz having the values $\rho_{q}=2.648 \times 10^{3} \mathrm{~kg} / \mathrm{m}^{3}$ and $u_{q}=2.947 \times$ $10^{11} \mathrm{~g} / \mathrm{cm} \cdot \mathrm{s}^{2}$, respectively.

However, there are still some inevitable drawbacks when using Kanazawa model for on-field measurement of liquid properties; that is, this method is difficult to be applied and spread widely in on-field testing because it is sample wasteful, requires complex instruments, and is difficult to operate. In addition, immersing the QCM in liquid will damp the amplitude, and the damping becomes seriously detrimental when immersed in highly viscous liquid, which will make the measurements hard or even causes the failure of oscillation.

This new mass-sensitivity-based approach presented in this paper could promote the application of QCMs in on-field testing of liquid viscosities and enable the measurement of highly viscous liquids. Thanks to the advantages of this new approach including simplicity of operation, saving reagent and time, real-time output, and label-free analysis, this novel approach will achieve a considerable potential application prospect in on-field measurements of liquids.

\section{Proposed Method}

The frequency shift caused by a localized or nonuniform mass attached on the QCM electrodes is given by [24]

$$
\Delta f=-\frac{1}{\pi r_{d}^{2}} \int_{0}^{2 \pi} \int_{0}^{r_{d}} S_{f}(r, \theta) m(r, \theta) r d r d \theta
$$

where $S_{f}(r, \theta)$ is the mass-sensitivity function, in hertz per kilogram; $m(r, \theta)$ is the effective added mass; $r_{d}$ is the radius for the mass deposit on the electrode; $r$ and $\theta$ are the polar coordinates of the point at which the mass is added.

The research of Josse et al. shows that the mass-sensitivity distribution of QCM devices can be represented by the following equation [25]:

$$
S_{f}(r, \theta)=\frac{|A(r)|^{2}}{2 \pi \int_{0}^{\infty} r|A(r)|^{2} d r} C_{f},
$$

where $C_{f}$ is the Sauerbrey sensitivity constant, with a value of $1.78 \times 10^{11} \mathrm{~Hz} \cdot \mathrm{cm}^{2} / \mathrm{kg} ; A(r)$ is the particle displacement amplitude function; and $r$ is the distance from the center.

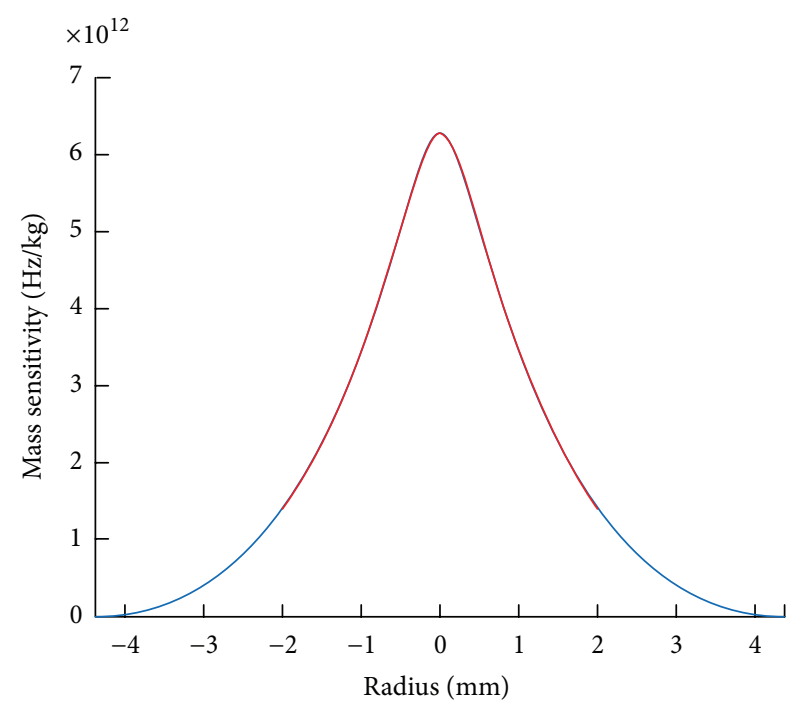

FIgure 1: Mass-sensitivity distribution for AT-cut $10 \mathrm{MHz}$ fundamental QCM. Red line represents the electrode region $(|r|<2 \mathrm{~mm})$ and blue line represents the nonelectrode region $(2 \mathrm{~mm}<|r|<$ $4.35 \mathrm{~mm})$.

The particle displacement amplitude function $A(r)$ is solution of the following particle displacement amplitude equation [26]:

$$
r^{2} \frac{\partial^{2} A}{\partial r^{2}}+r \frac{\partial A}{\partial r}+\frac{k_{i}^{2} r^{2}}{N} A=0
$$

where $k$ represents the wavenumber; $N$ is a constant determined by the elastic stiffness constants and the piezoelectric constants of crystal quartz. Since the particle displacement $A(r)$ and shear strain field are continuous at $r=m$ (where $m$ is the radius of electrode), according to these boundary conditions, the boundary equations of QCM with " $\mathrm{m}-\mathrm{m}$ " type electrode are obtained as [27]

$$
\begin{gathered}
\left.A^{E}\right|_{r=m}=\left.A^{U}\right|_{r=m}, \\
\left.\frac{\partial A^{E}}{\partial r}\right|_{r=m}=\left.\frac{\partial A^{U}}{\partial r}\right|_{r=m} .
\end{gathered}
$$

On the basis of these boundary conditions, $A(r)$ can be determined. And the mass-sensitivity function of QCM with " $\mathrm{m}-\mathrm{m}$ " type electrode is then determined [28].

Take two QCMs used in this study (AT-cut $10 \mathrm{MHz}$ fundamental QCM and AT-cut $10 \mathrm{MHz} 3$ rd overtone QCM), for example. The diameters of crystal and gold-electrode are $8.7 \mathrm{~mm}$ and $4 \mathrm{~mm}$, respectively. The thickness of the goldelectrode is $1000 \AA$. The profile of mass-sensitivity distribution can be obtained as shown in Figures 1 and 2, respectively.

As expected, the mass-sensitivity distribution of the two types of QCMs is an approximate Gaussian curve. The highest sensitivity point appears at the center of electrode $(r=$ 0 ) and then decreases exponentially as the distance from the center $(r)$ increases. So, within the electrode area, the 


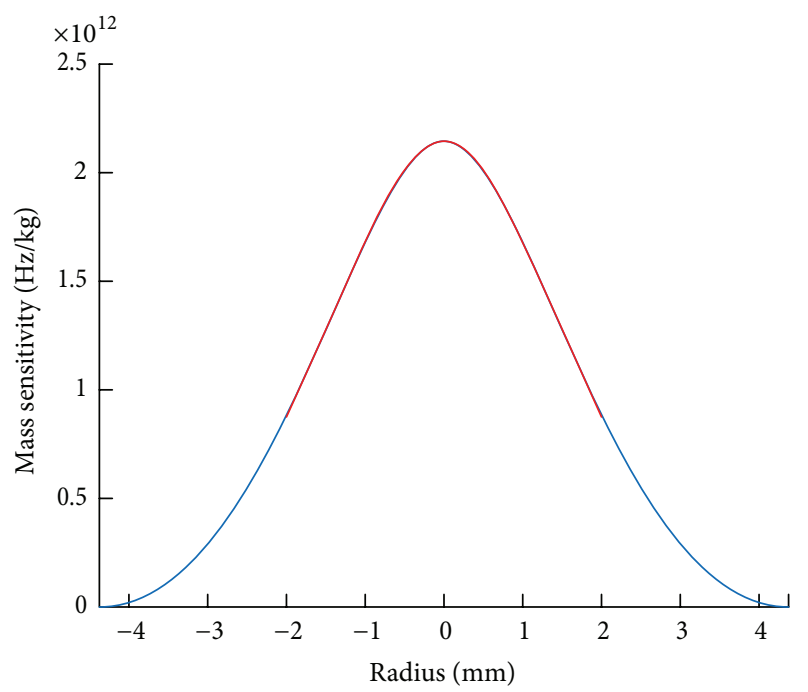

Figure 2: Mass-sensitivity distribution for AT-cut $10 \mathrm{MHz}$ 3rd overtone QCM. Red line represents the electrode region $(|r|<$ $2 \mathrm{~mm})$ and blue line represents the nonelectrode region $(2 \mathrm{~mm}<$ $|r|<4.35 \mathrm{~mm})$.

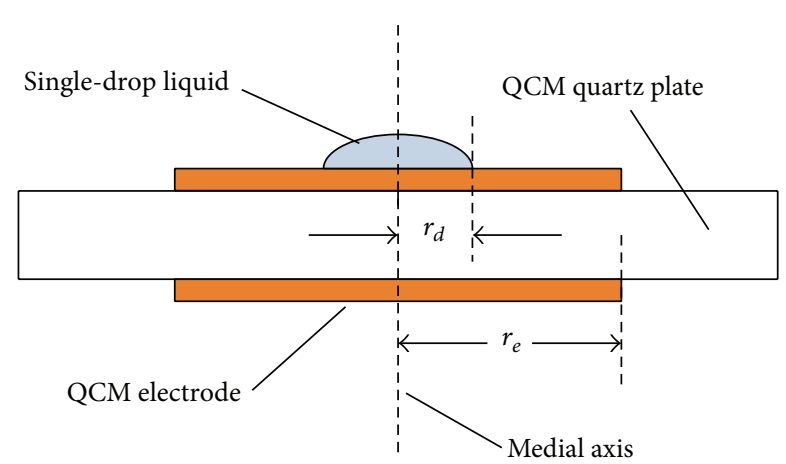

FIGURE 3: Schematic view of single-drop of liquid localized on the QCM.

mass sensitivity of the QCM can be described as a Gaussian function, like the following:

$$
S_{f}(r)=K e^{-x}, \quad\left(\text { where } x=\frac{\beta r^{2}}{r_{e}^{2}}\right),
$$

where $K$ is the highest value of sensitivity at the center of the electrode (for AT-cut $10 \mathrm{MHz}$ fundamental QCM $K=6.23$ $\times 10^{12}$ and for AT-cut $10 \mathrm{MHz} 3$ rd overtone QCM $K=2.02 \times$ $\left.10^{12}\right) ; r_{e}$ is the radius of the gold-electrode; and $\beta$ is a constant, for the two types of QCMs having the values 2.298 and 0.797, respectively.

As Figure 3 shows, when a small single-drop of liquid (in microliter level) loaded on the center of the electrode, the radius of single-drop much less than the electrode radius and their centers coincided; we can assume that $S_{f}(r)$ is changed a little within the range of $r<r_{d}$. As can be seen from Figures 1 and $2, S_{f}(r)$ is relatively smooth near the center of the electrode. In addition, the place loaded by liquid which has the highest mass sensitivity will contribute to lowering of the limit of detection.

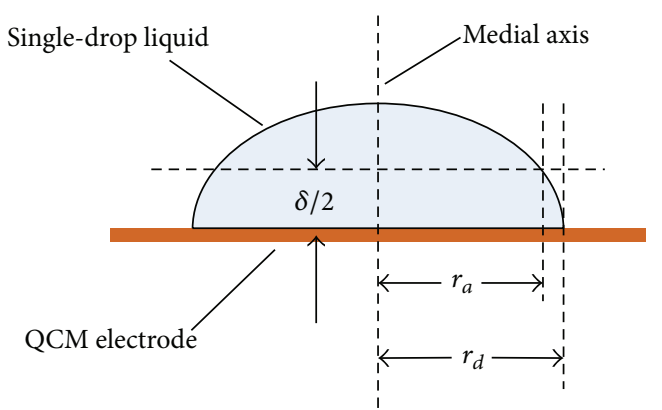

FIGURE 4: Schematic view of single-drop of liquid localized on the electrode.

The research of Hillier and Ward shows that the effective mass $\Delta m$ is related to the liquid decay length $\delta$ when the QCM is covered by a viscoelastic film [24]:

$$
\delta=\sqrt{\frac{\eta}{\pi f_{0} \rho}}
$$

where $\rho$ and $\eta$ are the density and viscosity of the liquid, respectively.

Based on the analysis above, as shown in Figure 4, the effective mass $\Delta m$ could be divided into $\Delta m_{1}$ (the effective mass of the liquid droplet contained within decay length $\delta / 2$ for base radius from center to $r_{a}$ ) and $\Delta m_{2}$ (the effective mass of the liquid droplet contained within decay length $\delta / 2$ for base radius from $r_{a}$ to $r_{d}$ ), and the frequency shift of QCM $\Delta f$ can be calculated as

$$
\begin{aligned}
\Delta f & =\Delta f_{1}+\Delta f_{2}, \\
\Delta f_{1} & =-\frac{2 \pi}{\pi r_{a}^{2}} \int_{0}^{r_{a}} K e^{-x} \Delta m_{1} r d r \\
\Delta f_{2} & =-\frac{2 \pi}{\pi\left(r_{d}^{2}-r_{a}^{2}\right)} \int_{r_{a}}^{r_{d}} K e^{-x} \Delta m_{2} r d r
\end{aligned}
$$

where $x=\beta r^{2} / r_{e}^{2}, \Delta m_{1}=\rho \pi r_{a}^{2} \delta / 2$, and $\Delta f_{1}$ and $\Delta f_{2}$ are the frequency shift caused by $\Delta m_{1}$ and $\Delta m_{2}$, respectively.

In general, the decay length of liquid is very small (in micrometer level), so $r_{d}-r_{a}$ is also in micrometer level; that is, $r_{d}-r_{a}$ is an infinitesimal. It is obvious that $\Delta m_{2} \approx$ $(1 / 2) \pi\left(r_{d}^{2}-r_{a}^{2}\right) \times \delta \times \rho$ is an infinitesimal of higher order than $\left(r_{d}-r_{a}\right)$. As a consequence, $\Delta f_{2}$ is very small and could be ignored. So $\Delta f$ can be calculated as

$$
\Delta f=-\pi \rho \delta \int_{0}^{r_{d}} K e^{-x} r d r
$$

where $x=\beta r^{2} / r_{e}^{2}$.

Equation (11) could be obtained through integration:

$$
\Delta f=-\frac{1}{2} \pi \rho \delta K \frac{r_{e}^{2}}{\beta}\left(1-e^{\widehat{x}}\right),
$$

where $\widehat{x}=-\beta r_{d}^{2} / r_{e}^{2}$. 


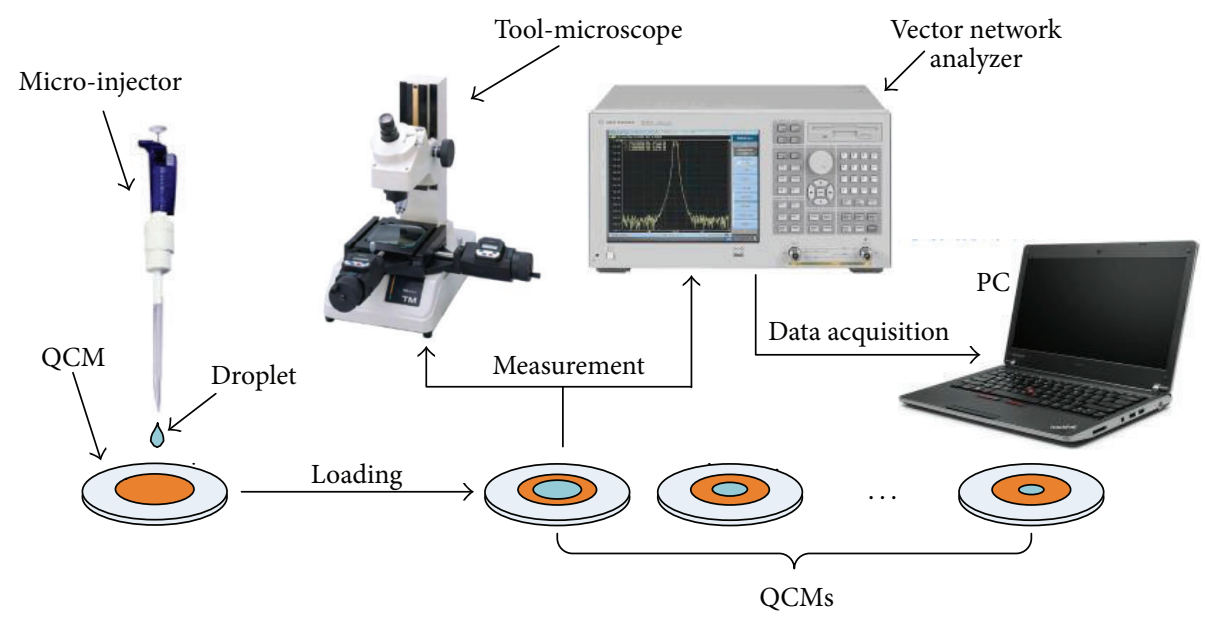

Figure 5: Schematic of experimental setup.

Since $r_{d} \ll r_{e}, \widehat{x}$ must be very small; then (12) is tenable:

$$
1-e^{\hat{x}} \approx-\left(\hat{x}+\frac{\hat{x}^{2}}{2}\right) .
$$

So (11) could be simplified to

$$
\Delta f=-\frac{1}{2} K r_{a}^{2} \sqrt{\frac{\pi \rho \eta}{f_{0}}}\left(1+\frac{\beta r_{d}^{2}}{2 r_{e}^{2}}\right) .
$$

In summary, considering that the density of liquids is usually known or can be measured easily and accurately using conventional methods, so the viscosity of liquids could be quickly and conveniently obtained by measuring the frequency shift of QCM and the radius of liquid drop according to (13).

\section{Experimental Results}

3.1. Experimental Setup. Figure 5 shows a schematic of the overall experimental setup. At room temperature (about $20^{\circ} \mathrm{C}$ ), a single-drop of sample liquid was loaded onto the center of the QCM electrode using a microinjector. Then the response of QCM was measured with a vector network analyzer (Agilent E5062A). And the diameter of the single-drop was measured with a tool-microscope (TM505). The microinjector was purchased from Shanghai Gaoge Industrial and Trading Co., Ltd. (Shanghai, China). The tool-microscope was purchased from Shanghai BM Optical Instruments and Manufacture Co., Ltd. (Shanghai, China).

It is worth adding that a high accuracy microscope was used to acquire high testing precision during experiments. But in practical on-field measurements, sophisticated high microscope is not needed; an ordinary magnifier loupe with scale can meet the measure requirements (with the magnifying power of about 100). Likewise, the expensive, sophisticated, and cumbersome vector network analyzer is also not needed and could be replaced by a small frequency meter. The computer used in these experiments is not necessary as well in practical on-field measurements; these simple calculations could be done by an ordinary calculator.

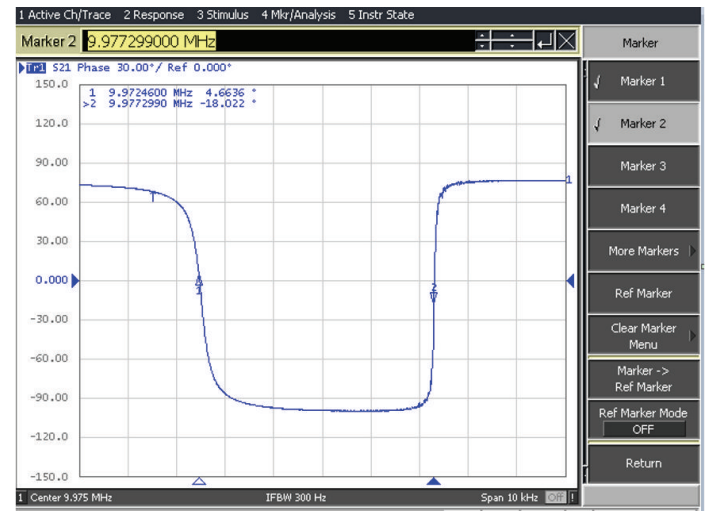

Figure 6: The response of AT-cut $10 \mathrm{MHz}$ fundamental QCM without loading.

3.2. The Measurement of Low Viscosity Liquid (Take Pure Water as an Example). Two types of QCMs were used in this study: AT-cut $10 \mathrm{MHz}$ fundamental QCM and AT-cut $10 \mathrm{MHz} 3 \mathrm{rd}$ overtone QCM. The only difference between them is the thickness of the crystal. The thickness of crystal of them is about $0.167 \mathrm{~mm}$ and $0.5 \mathrm{~mm}$, respectively. The thickness of the gold-electrode of them is $1000 \AA$. The diameter of crystal and gold-electrode is $8.7 \mathrm{~mm}$ and $4 \mathrm{~mm}$, respectively. They were purchased from Tongfang Guoxin Electronics Co., Ltd. (Hebei, China).

At room temperature (about $20^{\circ} \mathrm{C}$ ), about $0.5 \mu \mathrm{L}$ of pure water $\left(\eta=1.0022 \mathrm{mpa} \cdot \mathrm{s}\right.$ and $\left.\rho=0.9982 \times 10^{3} \mathrm{~kg} / \mathrm{m}^{3}\right)$ was loaded onto the center of QCM electrode using a microinjector. The response of AT-cut $10 \mathrm{MHz}$ fundamental QCM before and after loading is shown in Figures 6 and 7, respectively. The response of AT-cut $10 \mathrm{MHz} 3 \mathrm{rd}$ overtone QCM before and after loading is shown in Figures 8 and 9, respectively. The radius of the single-drop of water loaded on the two QCMs was measured as $0.57 \mathrm{~mm}$ and $0.56 \mathrm{~mm}$ using tool-microscope, respectively.

As can be seen from Figures 6 and 7, the resonant frequency of AT-cut $10 \mathrm{MHz}$ fundamental QCM declined 


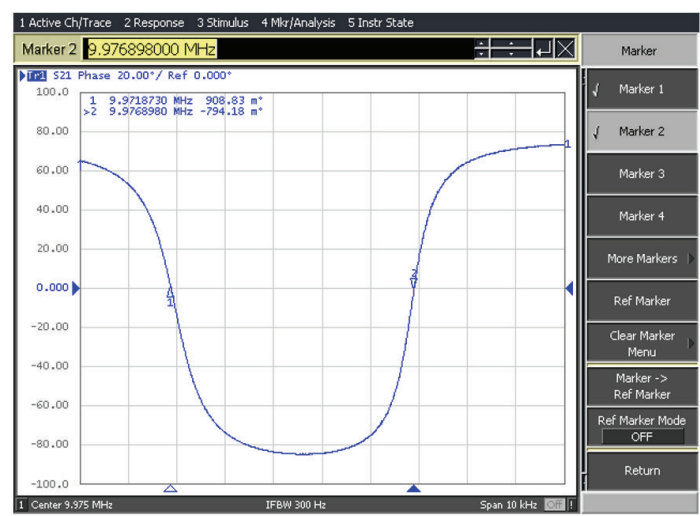

FIgURE 7: The response of AT-cut $10 \mathrm{MHz}$ fundamental QCM loaded with the single-drop of water.

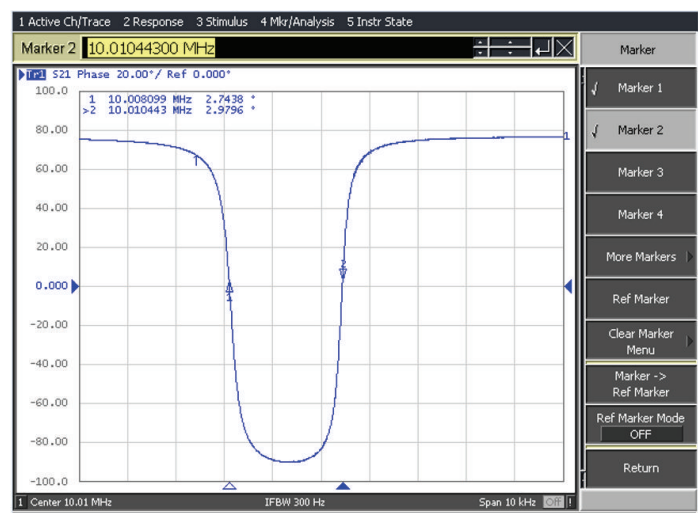

FIGURE 8: The response of AT-cut $10 \mathrm{MHz}$ 3rd overtone QCM without loading.

from 9.972460 MHz to 9.971873 MHz. So we can calculate the frequency shift of QCM $\Delta f=587 \mathrm{~Hz}$. Figures 8 and 9 showed that the resonant frequency of AT-cut $10 \mathrm{MHz} 3 \mathrm{rd}$ overtone QCM declined from 10.008099 MHz to $10.007919 \mathrm{MHz}$. So the frequency shift caused by the single-drop of water is $180 \mathrm{~Hz}(\Delta f=180 \mathrm{~Hz})$.

According to (13), we can figure out the theoretical frequency shift of each QCM: for AT-cut $10 \mathrm{MHz}$ fundamental QCM and AT-cut $10 \mathrm{MHz}$ 3rd overtone QCM, the theoretical frequency shift is $620 \mathrm{~Hz}$ and $186 \mathrm{~Hz}$, respectively. So the errors between theoretical values and experimental values are $5.6 \%$ and $3.3 \%$, respectively, and show that the experimental results agree well with the theory.

\subsection{The Measurement of High Viscosity Liquid (Take Organic} Reagent as an Example). Other two types of QCMs were used to test the organic reagent: QCM-4.5 and QCM-3; the only difference between them is the diameters of the gold-electrodes, which are $4.5 \mathrm{~mm}$ and $3 \mathrm{~mm}$, respectively. The electrode thicknesses of them are all about $1000 \AA$. The thicknesses and diameters of the quartz crystal wafers (with fundamental operating frequency of $10 \mathrm{MHz}$ ) are all about $0.167 \mathrm{~mm}$ and $8.7 \mathrm{~mm}$, respectively. They were purchased from Tongfang Guoxin Electronics Co., Ltd. (Hebei, China).

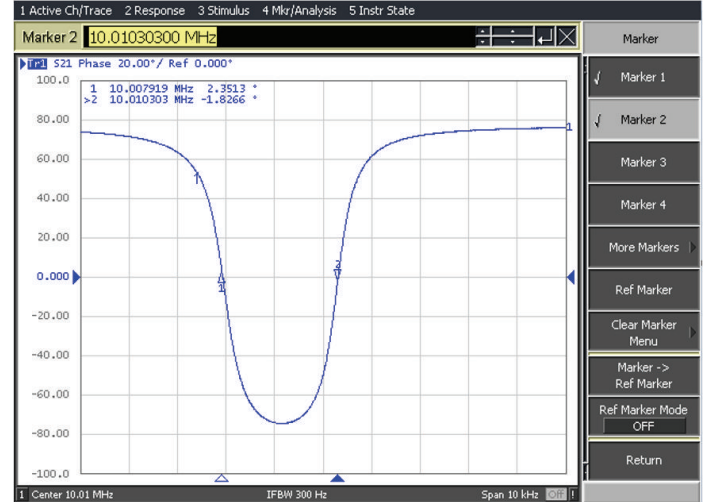

FIgURE 9: The response of AT-cut $10 \mathrm{MHz}$ 3rd overtone QCM loaded with the single-drop of water.

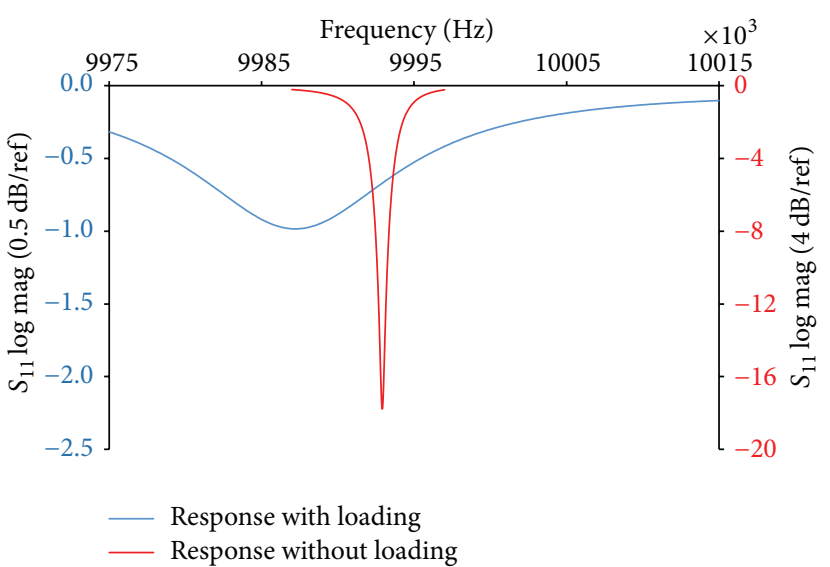

FIGURE 10: The responses of QCM- 4.5 without and with the singledrop loading which has a radius of $0.94 \mathrm{~mm}$.

The organic reagents were purchased from BP Corporate Introduction, and the density and viscosity of it are $\rho=$ $0.842 \times 10^{3} \mathrm{~kg} / \mathrm{m}^{3}$ and $\eta=110 \mathrm{mpa} \cdot \mathrm{s}$ at room temperature, respectively.

As shown in Figure 5, at room temperature (about $20^{\circ} \mathrm{C}$ ), a drop of organic reagent was loaded near the center of the electrode of QCM using a microinjector; then the response of QCM and the diameter of single-drop were measured and recorded. Ten experiments were carried out with different sizes of droplets using two types of QCMs (five experiments for each type of QCM, and each experiment was carried out using a new QCM).

Take the second experiment (using a QCM-4.5) and sixth experiment (using a QCM-3), for example. In the second experiment, the responses of the QCM-4.5 without and with the single-drop loading which has a radius of $0.94 \mathrm{~mm}$ are shown in Figure 10. The resonant frequency of the QCM-4.5 declined from $9992915 \mathrm{~Hz}$ to $9987602 \mathrm{~Hz}$, so the frequency shift can be calculated as $\Delta f=5313 \mathrm{~Hz}$. In the sixth experiment, the response of the QCM-3 without and with the single-drop loading which has a radius of $0.42 \mathrm{~mm}$ is shown in Figure 11. The resonant frequency of the QCM-3 


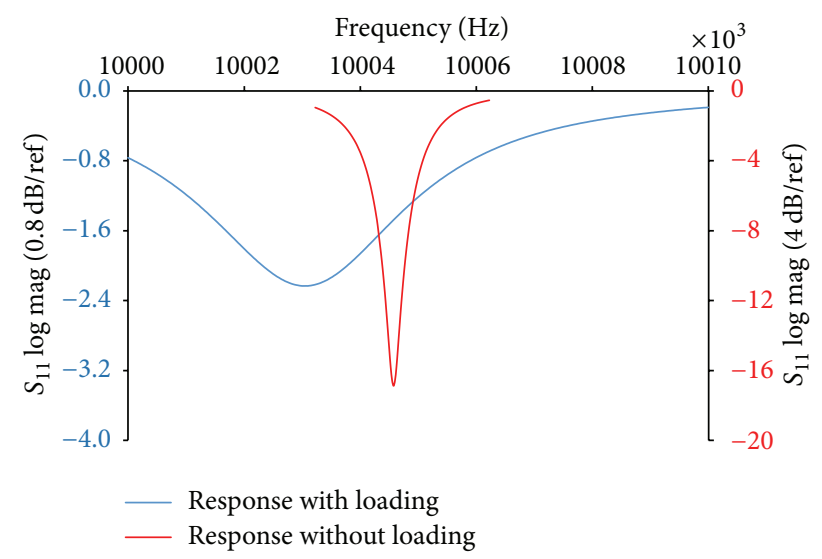

FIGURE 11: The responses of QCM-3 without and with the singledrop loading which has a radius of $0.42 \mathrm{~mm}$.

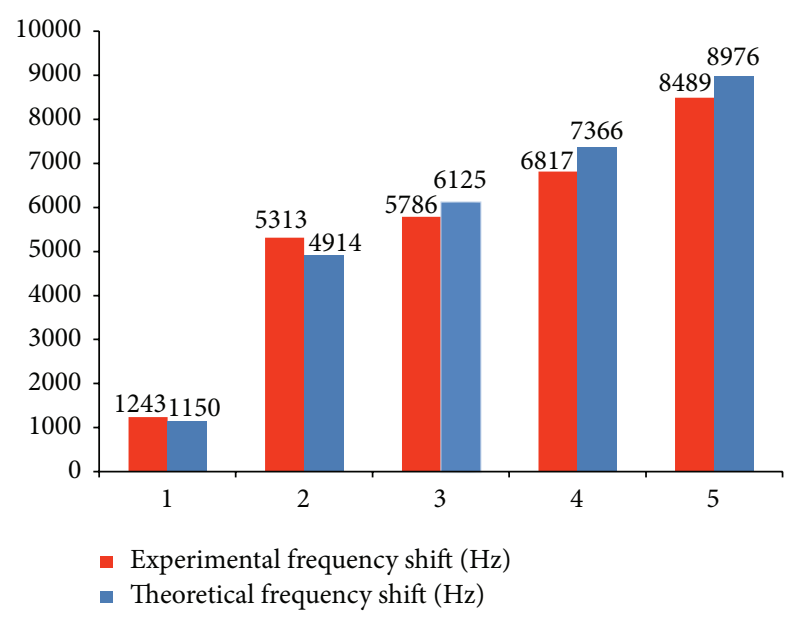

FIgURE 12: Comparisons between experimental and theoretical values of first five experiments (using QCM-4.5).

declined from $10004584 \mathrm{~Hz}$ to $10003180 \mathrm{~Hz}$, so the frequency shift can be calculated as $\Delta f=1404 \mathrm{~Hz}$.

The results of all ten experiments are as shown in Table 1 , where $r_{d}$ is the radius of droplets and $\Delta f$ and $\Delta f_{t}$ are the experimental and theoretical frequency shift, respectively. Moreover, the errors between experimental and theoretical values were calculated according to $E_{e}=\left(\Delta f-\Delta f_{t}\right) / \Delta f_{t}$. The QCM used in the first five experiments is QCM-4.5 and QCM used in the last five experiments is QCM-3. The comparisons between experimental and theoretical values are illustrated in Figure 12 (using QCM-4.5) and Figure 13 (using QCM-3) according to experiment sequence number as abscissa and frequency shift (in $\mathrm{Hz}$ ) as coordinate.

As can be seen from Table 1 and Figures 12 and 13, to both types of QCMs, the absolute values of errors between theoretical and experimental frequency shift are all less than $8.2 \%$, so the experimental data can be thought of in accordance with the theoretical inferences.

3.4. Error Analysis. There are several reasons that account for the errors between experimental and theoretical values.
TABLE 1: Experiment results and theoretic values. The QCM used in the first five experiments is QCM-4.5 and QCM used in the last five experiments is QCM-3.

\begin{tabular}{lcccc}
\hline Number & $r_{d}(\mathrm{~mm})$ & $\Delta f(\mathrm{~Hz})$ & $\Delta f_{t}(\mathrm{~Hz})$ & Error \\
\hline 1 & 0.49 & 1243 & 1150 & $+4.4 \%$ \\
2 & 0.94 & 5313 & 4914 & $+8.1 \%$ \\
3 & 1.03 & 5786 & 6125 & $-5.5 \%$ \\
4 & 1.11 & 6817 & 7366 & $-7.5 \%$ \\
5 & 1.20 & 8489 & 8967 & $-5.3 \%$ \\
\hline 6 & 0.42 & 1404 & 1513 & $-7.2 \%$ \\
7 & 0.62 & 3348 & 3576 & $-5.4 \%$ \\
8 & 0.76 & 6115 & 5764 & $+6.1 \%$ \\
9 & 0.81 & 6316 & 6727 & $-6.2 \%$ \\
10 & 0.85 & 7017 & 7576 & $-7.4 \%$ \\
\hline
\end{tabular}

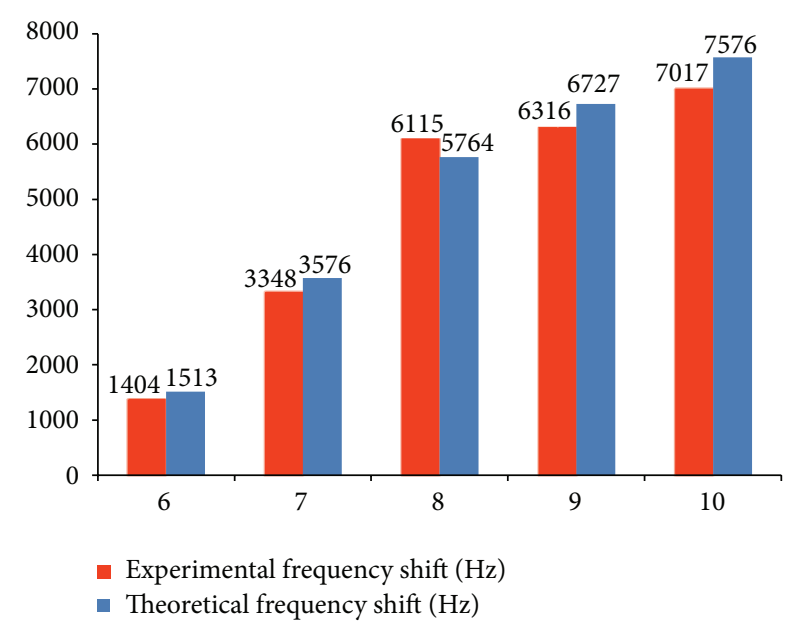

FIGURE 13: Comparisons between experimental and theoretical values of last five experiments (using QCM-3).

(a) Operation errors: it is inevitable to have operation error in the process of experiments, such as the fact that the droplet cannot be loaded on the absolute center of the electrode accurately.

(b) Measurement errors: both of systematic errors of toolmicroscope and visual perception errors of human will cause measurement error during the measurements of droplet radius with tool-microscope.

(c) Theoretic errors: in the above process of theoretic deriving, approximate treatments are made in (7), (11), and (13) to simplify the calculations. By the verification of error analysis, it should be noted that the errors caused by approximate treatments in theory are less than $3 \%$, and the errors will decrease with the reduction of the droplet radius.

(d) Disturbing errors: disturbance of environments such as humidity, temperature, and airflow will cause disturbing errors inevitably.

(e) In addition, the vibration mode of quartz crystal is likely to be affected by the load distribution, especially the asymmetric or heterogeneous load distribution, 
and then impact the sensitivity distribution. And this issue is worthy of further research.

If the above problems can be solved, the errors between experimental and theoretical values could be controlled at an ideal level. And it leads to the direction of future study of this problem.

\section{Conclusions}

A novel mass-sensitivity-based method to measure liquid viscosities using a QCM is introduced in this paper, and the relative relationship between the frequency shift and the viscosity of sample liquid is also revealed. Lots of scientific studies and practical applications need to measure liquid viscosities, and this new method has broad prospects for application in on-field testing due to its advantages including reagent-saving, wide response spectrum, simplicity of operation, real-time output, and label-free analysis. At the same time, it could enhance the application of QCMs in various research and industrial fields.

\section{Conflict of Interests}

The authors declare that there is no conflict of interests regarding the publication of this paper.

\section{References}

[1] P. Kashulines, S. S. H. Rizvil, P. Harriott, and J. A. Zollweg, "Viscosities of fatty acids and methylated fatty acids saturated with supercritical carbon dioxide," Journal of the American Oil Chemists Society, vol. 68, no. 12, pp. 912-921, 1991.

[2] B. Calvignac, E. Rodier, J.-J. Letourneau, P. Vitoux, C. Aymonier, and J. Fages, "Development of an improved falling ball viscometer for high-pressure measurements with supercritical $\mathrm{CO}_{2}$," Journal of Supercritical Fluids, vol. 55, no. 1, pp. 96-106, 2010.

[3] M. Mark, K. Häusler, J. Dual, and W. H. Reinhart, "Oscillating viscometer-evaluation of a new bedside test," Biorheology, vol. 43, no. 2, pp. 133-146, 2006.

[4] V. S. K. Prasad, K. Balasubramaniam, E. Kannan, and K. L. Geisinger, "Viscosity measurements of melts at high temperatures using ultrasonic guided waves," Journal of Materials Processing Technology, vol. 207, no. 1-3, pp. 315-320, 2008.

[5] K. M. M. Kabir, S. J. Ippolito, G. I. Matthews, S. B. Abd Hamid, Y. M. Sabri, and S. K. Bhargava, "Determining the optimum exposure and recovery periods for efficient operation of a QCM based elemental mercury vapor sensor," Journal of Sensors, vol. 2015, Article ID 727432, 7 pages, 2015.

[6] S. K. Vashist and P. Vashist, "Recent advances in quartz crystal microbalance-based sensors," Journal of Sensors, vol. 2011, Article ID 571405, 13 pages, 2011.

[7] Z. Parlak, C. Biet, and S. Zauscher, "Decoupling mass adsorption from fluid viscosity and density in quartz crystal microbalance measurements using normalized conductance modeling," Measurement Science \& Technology, vol. 24, no. 8, Article ID 085301, 2013.

[8] K. Kanazawa and N.-J. Cho, "Quartz crystal microbalance as a sensor to characterize macromolecular assembly dynamics," Journal of Sensors, vol. 2009, Article ID 824947, 17 pages, 2009.
[9] J. Rabe, S. Büttgenbach, J. Schröder, and P. Hauptmann, "Monolithic miniaturized quartz microbalance array and its application to chemical sensor systems for liquids," IEEE Sensors Journal, vol. 3, no. 4, pp. 361-368, 2003.

[10] J.-H. Choi, K. K. Kanazawa, and N.-J. Cho, "Effect of a nonNewtonian load on signature $S_{2}$ for quartz crystal microbalance measurements," Journal of Sensors, vol. 2014, Article ID 373528, 8 pages, 2014.

[11] A. Itoh and M. Ichihashi, "Separate measurement of the density and viscosity of a liquid using a quartz crystal microbalance based on admittance analysis (QCM-A)," Measurement Science and Technology, vol. 22, no. 1, Article ID 015402, 2011.

[12] M. I. Newton, S. Atherton, R. H. Morris et al., "Low-cost QCM sensor system for screening semen samples," Journal of Sensors, vol. 2010, Article ID 326365, 5 pages, 2010.

[13] I. D. Avramov, "A quartz crystal sensor oscillator appropriate for highly viscous liquid measurements," Measurement Science \& Technology, vol. 20, no. 12, Article ID 124006, 2009.

[14] M. H. Wang, S. L. Liu, Y. C. Zhang et al., "Graphene nanostructures with plasma polymerized allylamine biosensor for selective detection of mercury ions," Sensors and Actuators B: Chemical, vol. 203, pp. 497-503, 2014.

[15] Y. Xie, Y. Jin, Y. Huang, G. Liu, and R. Zhao, "Highly selective piezoelectric sensor for lead(II) based on the lead-catalyzed release of gold nanoparticles from a self-assembled nanosurface," Microchimica Acta, vol. 181, no. 13-14, pp. 1521-1527, 2014.

[16] Z.-P. Yang and C.-J. Zhang, "Designing of MIP-based QCM sensor for the determination of $\mathrm{Cu}(\mathrm{II})$ ions in solution," Sensors and Actuators B: Chemical, vol. 142, no. 1, pp. 210-215, 2009.

[17] H. F. El-Sharif, H. Aizawa, and S. M. Reddy, "Spectroscopic and quartz crystal microbalance (QCM) characterisation of protein-based MIPs," Sensors and Actuators B: Chemical, vol. 206, pp. 239-245, 2015.

[18] D. Lee and T. Cui, "Layer-by-layer self-assembled single-walled carbon nanotubes based ion-sensitive conductometric glucose biosensors," IEEE Sensors Journal, vol. 9, no. 4, pp. 449-456, 2009.

[19] Y. M. Sabri, S. J. Ippolito, A. P. O’Mullane, J. Tardio, V. Bansal, and S. K. Bhargava, "Creating gold nanoprisms directly on quartz crystal microbalance electrodes for mercury vapor sensing," Nanotechnology, vol. 22, no. 30, Article ID 305501, 2011.

[20] A. Mirmohseni, R. Pourata, and M. Shojaei, "Application of molecularly imprinted polymer for determination of glucose by quartz crystal nanobalance technique," IEEE Sensors Journal, vol. 14, no. 8, pp. 2807-2812, 2014.

[21] E. Juzeliunas, "Quartz crystal microgravimetry-fify years of application and new challenges," Chemija, vol. 20, no. 4, pp. 218225, 2009.

[22] G. Sauerbrey, "Use of quartz vibration for weighing thin films on a microbalance," Zeitschrift für Physik, vol. 155, no. 2, pp. 206212, 1959.

[23] K. K. Kanazawa and J. G. Gordon II, “The oscillation frequency of a quartz resonator in contact with liquid," Analytica Chimica Acta, vol. 175, pp. 99-105, 1985.

[24] A. C. Hillier and M. D. Ward, "Scanning electrochemical mass sensitivity mapping of the quartz crystal microbalance in liquid media," Analytical Chemistry, vol. 64, no. 21, pp. 2539-2554, 1992.

[25] F. Josse, Y. Lee, S. J. Martin, and R. W. Cernosek, "Analysis of the radial dependence of mass sensitivity for modified-electrode 
quartz crystal resonators," Analytical Chemistry, vol. 70, no. 2, pp. 237-247, 1998.

[26] J. Gao, X. Huang, and Y. Wang, "The modified design of ring electrode quartz crystal resonator for uniform mass sensitivity distribution," IEEE Transactions on Ultrasonics, Ferroelectrics, and Frequency Control, vol. 60, no. 9, pp. 2031-2034, 2013.

[27] S. K. R. S. Sankaranarayanan, R. Singh, and V. Bhethanabotla, "Computational design of quartz crystal nanobalance for uniform sensitivity distribution," in Proceedings of the 9th IEEE Sensors Conference (SENSORS '10), pp. 1883-1886, Kona, Hawaii, USA, November 2010.

[28] S. J. Martin, V. E. Granstaff, and G. C. Frye, "Characterization of a quartz crystal microbalance with simultaneous mass and liquid loading," Analytical Chemistry, vol. 63, no. 20, pp. 22722281, 1991. 


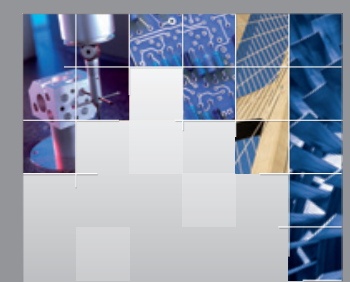

\section{Enfincering}
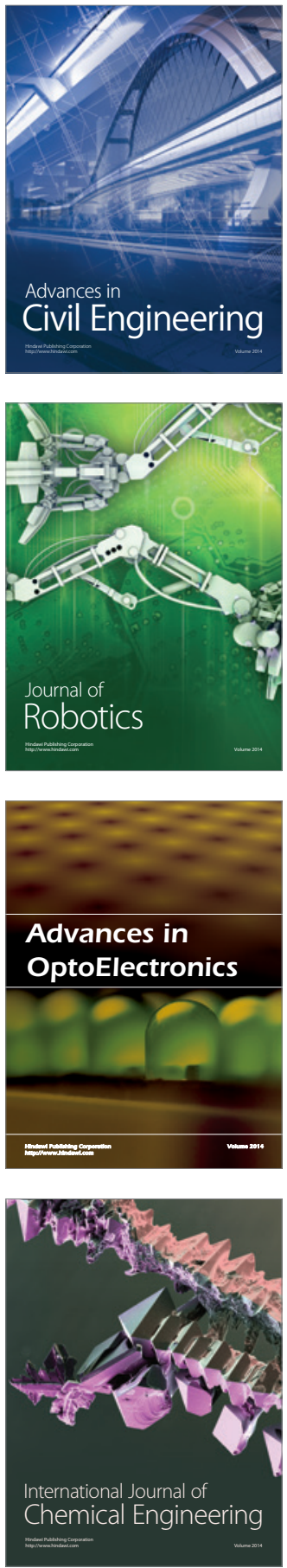

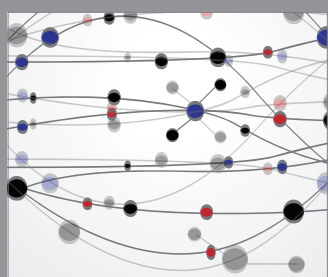

The Scientific World Journal

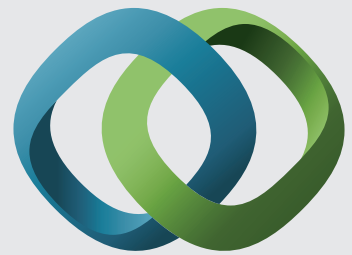

\section{Hindawi}

Submit your manuscripts at

http://www.hindawi.com
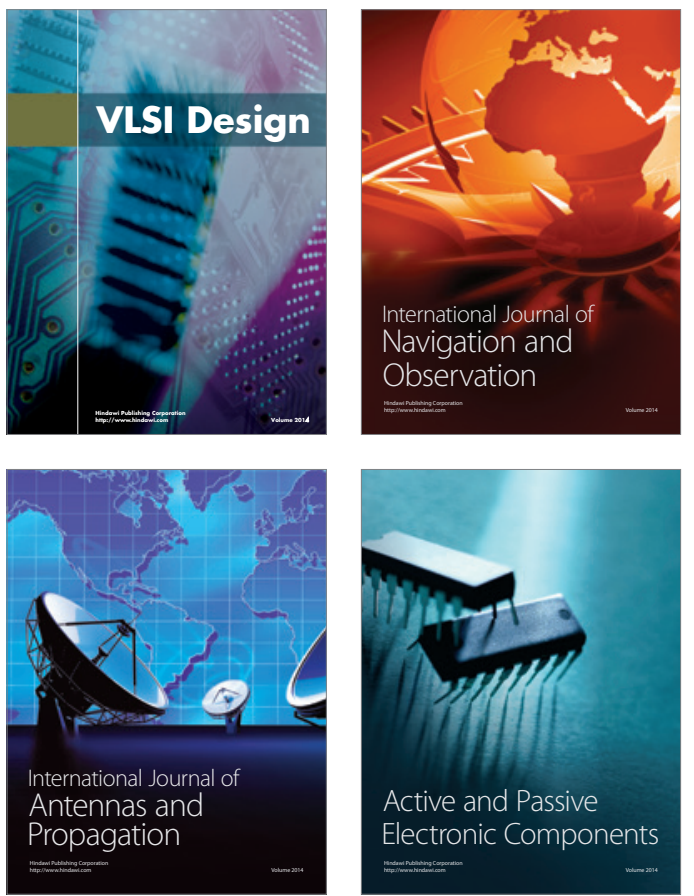
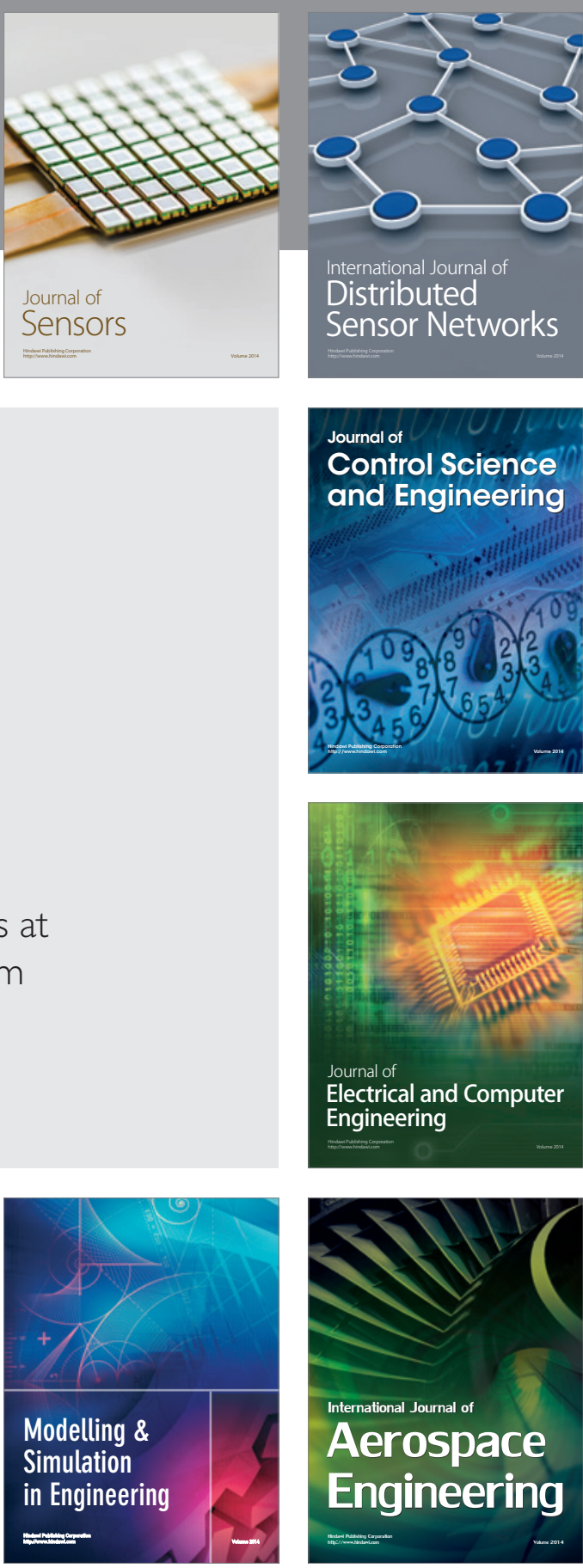

International Journal of

Distributed

Sensor Networks

Journal of

Control Science

and Engineering
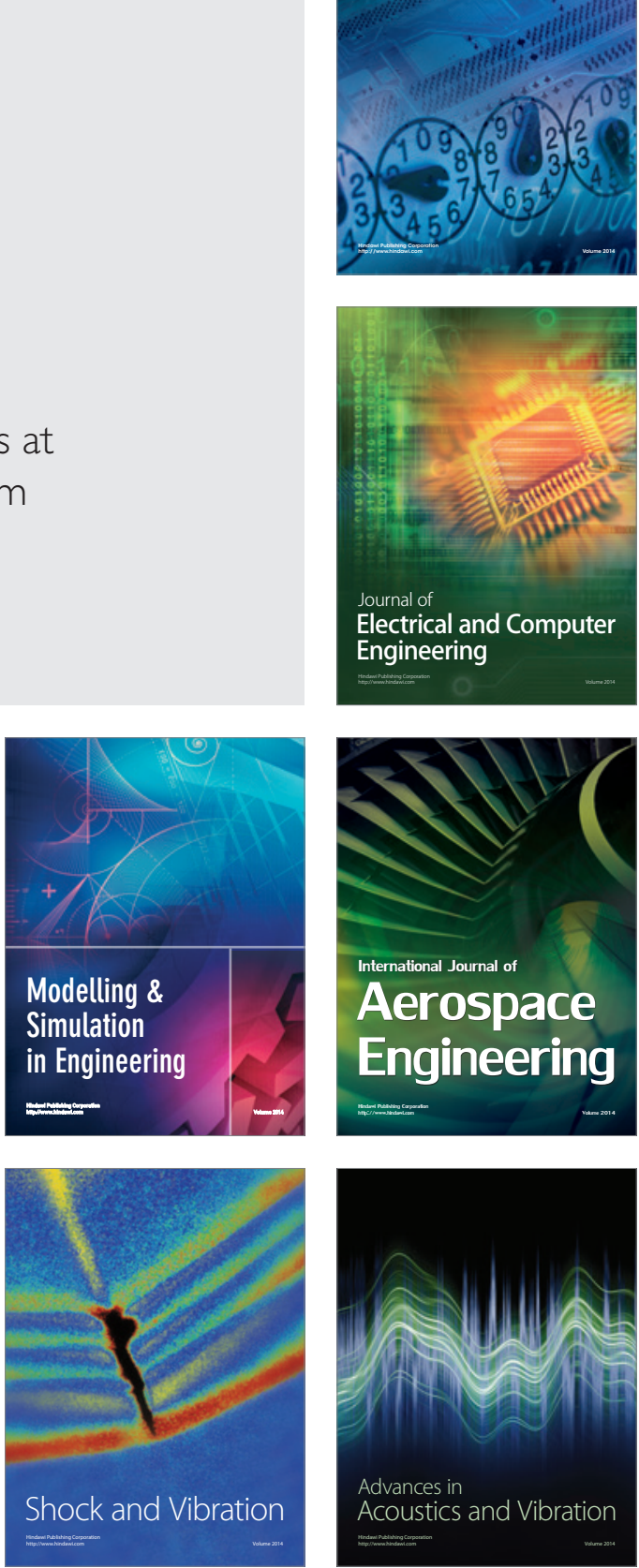